medical intervention and prevention of further progression of disease through improved management of these conditions.

\section{S131 WHAT PROPORTION OF THE UK POPULATION WOULD BE ELIGIBLE FOR CT SCREENING FOR LUNG CANCER ACCORDING TO VARIOUS PROPOSED INCLUSION CRITERIA?}

K Gracie, M Kennedy, J Robson, M Callister. Leeds Teaching Hospitals NHS Trust, Leeds, UK

\subsection{6/thoraxjnl-2016-209333.137}

Introduction Low dose CT screening reduced lung cancer mortality by $20 \%$ in the National Lung Screening Trial (NLST) using eligibility criteria of age 55-74 yrs, $\geq 30$ pack year smoking history, and quit time $<15$ years. The US Preventative Services Task Force (USPSTF) has proposed using the NLST criteria extending the upper age limit to 80 years. Alternative proposed eligibility criteria use thresholds from composite risk prediction scores such as a $1.51 \%$ lung cancer risk over 6 years using the PLCO $_{M 2012}$ model (derived from the Prostate Lung Colorectal and Ovarian Study) and a 5\% lung cancer risk over 5 years using the Liverpool Lung Project (LLP) model (used in the UK Lung Screening trial). We sought to compare the proportions of patients in the UK who would be eligible for screening according to these criteria.

Methods We commissioned an anonymous telephone survey in Yorkshire (Hull, Leeds and Wakefield) to collect parameters to calculate lung cancer risk ( PLCO $_{\mathrm{M} 2012}$ and LLPv.2) and likelihood of participation in a future programme. Index of multiple deprivation (IMD) was recorded based on postcode and used to ensure a representative cohort. No patient identifiable information was entered into the research database.
Results 2,424 persons 55-80 years agreed to participate in the telephone survey, of which 1,335 were ever-smokers. The proportion of patients $(95 \% \mathrm{CI})$ eligible according to various criteria were as follows: NLST 11.9\% (10.6\%-13.2\%), USPSTF $13.3 \%$ (12.0\%-14.7\%), PLCO $_{\mathrm{M} 2012} \geq 1.51 \% \quad 20.7 \% \quad(19.1 \%-22.3 \%)$ and LLP $\geq 5 \% 15.8 \%(14.4 \%-17.3 \%)$. The proportions eligible by USPSTF, PLCO and LLP criteria by IMD and age cohort are shown in Figure 1. When asked how likely they would be to attend an NHS lung cancer screening programme, 62.6\% indicated 'very likely'. This proportion was similar between those eligible for screening by any criteria and those not $(62.5 \%$ and $62.7 \%$ respectively) and current and ex-smokers $(61.4 \%$ and $63.0 \%$ respectively).

Discussion The proportions of the population eligible for screening differ considerably between various eligibility criteria, and according to deprivation and age. The criteria selected to determine screen-eligibility in a future national screening programme will have a significant impact on the cost and cost-effectiveness of such a programme.

\section{S132 A RANDOMISED CONTROLLED STUDY OF LUNG CANCER SCREENING IN SCOTLAND USING THE DETECTION OF AUTOANTIBODIES TO TUMOUR ANTIGENS (EARLYCDT-LUNG TEST)}

${ }^{1} \mathrm{~A}$ Dorward, ${ }^{1} \mathrm{~F}$ Frances Mair, ${ }^{2} \mathrm{~F}$ Sullivan, ${ }^{3} \mathrm{~K}$ Vedhara, ${ }^{3} \mathrm{D}$ Kendrick, ${ }^{4} \mathrm{~S}$ Treweek, ${ }^{5} \mathrm{C}$ McCowan, ${ }^{5} \mathrm{~A}$ McConnachie, ${ }^{1} \mathrm{M}$ Sproule, ${ }^{5} \mathrm{~A}$ Briggs, ${ }^{4} \mathrm{~L}$ Ritchie, ${ }^{1} \mathrm{R}$ Milroy, ${ }^{6} \mathrm{~T}$ Taylor, ${ }^{7} \mathrm{R}$ Littleford, ${ }^{8} \mathrm{D}$ Brewster, ${ }^{6} \mathrm{~S}$ Schembri. ${ }^{7} \mathrm{NHS}$ Greater Glasgow and Clyde, Glasgow, UK; ${ }^{2}$ University of Toronto, Toronto, Canada; ${ }^{3}$ University of Nottingham, Nottingham, UK; ${ }^{4}$ University of Aberdeen, Aberdeen, UK; ${ }^{5}$ University of Glasgow, Glasgow, UK; ${ }^{6} \mathrm{NHS}$ Tayside, Dundee, UK; ${ }^{7} T C T U$, University of Dundee, Dundee, UK; ${ }^{8}$ Scottish Cancer Registry, Edinburgh, UK

10.1136/thoraxjnl-2016-209333.138

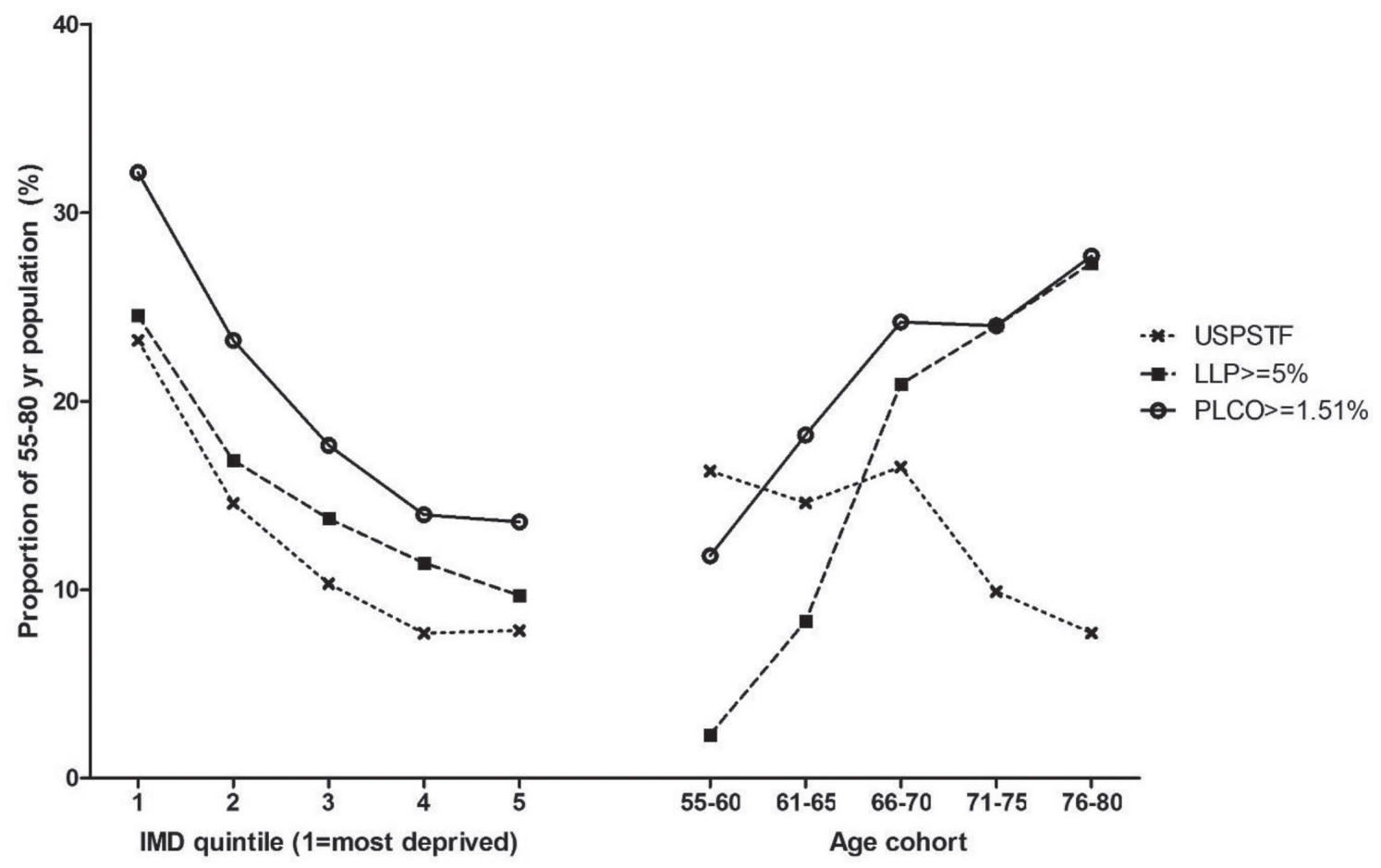

Abstract S131 Figure 1 The proportion of 55-80 year old population eligible for screening by various criteria by IMD quintile and age 
Background EarlyCDT ${ }^{\circledR}$-Lung Test detects autoantibodies to abnormal cell surface proteins from the early stages of lung cancer with a specificity of $93 \%$. This may allow earlier tumour detection thus altering prognosis.

The primary research question is: Does using the EarlyCDT ${ }^{\circledR}$ Lung Test to identify those at high risk of lung cancer, followed by xray and CT scanning in the test positive group, reduce the incidence of patients with late-stage lung cancer (III and IV) or unclassified presentation (U) at diagnosis, compared to standard practice? Recruitment was completed in June 2016 with 12,018 subjects randomised.

Methods A RCT in Scotland recruiting from the most socially disadvantaged quintiles. Adults aged 50 to 75 (ECOG 0-2) who were at high risk for lung cancer ( $>20$ pack years or relevant family history) were eligible. The intervention was the EarlyCDT ${ }^{\circledR}$-Lung Test, followed by chest xray and CT in those with a positive result. The comparator is standard clinical practice in the UK. The primary outcome is the difference, after 24 months, between the rates of patients with stage III, IV or unclassified lung cancer at diagnosis in test $\mathrm{v}$ no-test group. Secondary outcomes include: all-cause mortality; cancer specific mortality; a range of morbidity outcomes; cost-effectiveness and measures examining the psychological and behavioural consequences of screening.

Participants with a positive test result had an initial chest xray which was used to determine the urgency and the need for contrast in the initial screening CT. Those in whom the initial CT scan did not lead to a lung cancer diagnosis were offered biannual chest CTs for 24 months. Participants who are found to have lung cancer will be followed-up to assess both time to diagnosis and stage of disease at diagnosis.

Results $575 / 6120(9.8 \%)$ of the test group had a positive test with 207 found to have lung nodules $>8 \mathrm{~mm}, 16$ cancers have been detected so far, 12 of which are at early stage. Eleven have abnormalities undergoing current investigation. At this stage of the trial we have no outcome data for the comparison group. Conclusion The study will determine EarlyCDT-Lung test's clinical and cost effectiveness.

\section{Respiratory Science}

\section{S133 INVESTIGATING GENOME WIDE DNA METHYLATION IN AIRWAY AND PARENCHYMAL FIBROBLASTS FROM HEALTHY INDIVIDUALS AND INDIVIDUALS WITH COPD}

${ }^{1} \mathrm{RL}$ Clifford, ${ }^{2} \mathrm{~N}$ Fishbane, ${ }^{1} \mathrm{P}$ Rajasekar, ${ }^{3} \mathrm{AJ}$ Fisher, ${ }^{4} \mathrm{MS}$ Kobor, ${ }^{1} \mathrm{AJ}$ Knox, ${ }^{2} \mathrm{TL}$ Hackett. ${ }^{1}$ Division of Respiratory Medicine and Nottingham Respiratory Research Unit, University of Nottingham, Nottingham, UK; '2University of British Columbia, Centre for Heart Lung Innovation, Vancouver, Canada; ${ }^{3}$ Department of Respiratory Medicine, Newcastle Upon Tyne Hospitals NHS Foundation Trust, Newcastle Upon Tyne, UK; ${ }^{4}$ Centre for Molecular Medicine and Therapeutics, Child and Family Research Institute, Vancouver, Canada

\subsection{6/thoraxjnl-2016-209333.139}

Rationale Lung fibroblasts are implicated in respiratory disease pathology including chronic obstructive pulmonary disease (COPD). Phenotypic differences between fibroblasts isolated from the airway versus the parenchyma have been described but no studies have compared the cell types on a genome wide scale. DNA methylation is a reversible modification of the DNA structure with the ability to affect cell function via the alteration of gene expression. Here we compared genome wide DNA methylation profiles from airway and parenchymal fibroblasts and assessed modification to these profiles in cells isolated from individuals with COPD.

Methods DNA was isolated from parenchymal and airway fibroblasts at passage 4, and bisulphite treated. Site specific, quantitative genome wide methylation was determined using the Illumina 450K Infinium Methylation BeadChip array. Linear modelling and DMRcate functions identified differentially methylated sites and regions respectively between airway and parenchymal fibroblasts isolated from individuals with normal lung function versus those with COPD.

Results 3980 CpG (methylation) sites significantly differed after Bonferroni correction between airway and parenchymal fibroblasts isolated from healthy individuals. These sites had a broad distribution of effect size, with $240 \mathrm{CpG}$ sites displaying a difference in methylation of $>50 \%$. 78 of these sites validated in a second cohort of 7 sets of paired airway and parenchymal fibroblasts isolated from the same individual. There was genomic proximity to these sites and DMRcate was used to refine the individual $\mathrm{CpG}$ sites to 5 regions of interest associated with 5 genes; HLX, TWIST1, CREB5, SKAP2 and PRDM16. Differences in methylation were less pronounced when comparing cells isolated from healthy individuals to those with COPD. In airway fibroblasts 47 DMRcate regions were identified with a maximum difference in methylation of at least 20\%. In parenchymal fibroblasts 3 DMRcate regions were identified with a maximum difference in methylation of at least $20 \%$.

Conclusions DNA methylation profiles are significantly different between airway and parenchymal fibroblasts but only small modifications are associated with COPD. Future work will focus on validating a methylation based markers of parenchymal versus airway fibroblasts and associating our differential observations with gene/protein expression.

\section{S134 HOW SPECIFIC ARE FLUOROGENIC SUBSTRATES DESIGNED TO ANALYSE ACTIVE PROTEASE BIOMARKERS OF RESPIRATORY DISEASE?}

${ }^{1} \mathrm{CM}$ Robb, ${ }^{1} \mathrm{TEG}$ Ferguson, ${ }^{1} \mathrm{KL}$ Moffitt, ${ }^{2} \mathrm{DG}$ Downey, ${ }^{1} \mathrm{DF}$ McCafferty, ${ }^{1} \mathrm{D}$ Ribeiro, ${ }^{3} \mathrm{SL}$ Martin, ${ }^{1} \mathrm{~B}$ Walker. ${ }^{1}$ ProAxsis, Belfast, UK; ${ }^{2}$ Belfast Health and Social Care Trust, Belfast, UK; ${ }^{3}$ Queens University Belfast, Belfast, UK

\subsection{6/thoraxjnl-2016-209333.140}

Introduction Active proteases, such as neutrophil elastase (NE) and matrix metalloproteinases (MMPs), have been established as inflammatory biomarkers in lung diseases such as cystic fibrosis (CF), chronic obstructive pulmonary disease (COPD) and pulmonary fibrosis. Therefore, biological samples collected during clinical investigations are often analysed using fluorogenic substrates to determine protease activity and identify correlations with clinical and/or demographic parameters. Due to the nature of these diseases, samples often contain numerous active proteases from both human and bacterial origins which collectively have significant substrate crossover. This study investigates the ability of fluorogenic substrates to distinguish between proteases in complex clinical samples and provide an indication of the predictive capability of this assay type.

Methods Expectorated sputum was randomly collected from patients with CF who were hospitalised for an acute exacerbation. Samples were processed within 30 minutes of collection, and the aqueous sol recovered, pooled, aliquoted and stored at $-80^{\circ} \mathrm{C}$ until analysis. The capacity of sputum proteases to hydrolyse fluorogenic substrates with and without the presence of inhibitors specific for serine (multiple subclasses), metallo and 